\title{
Robust Initialization of Rigorous Process Simulations of Multiple Dividing Wall Columns via $V_{\text {min }}$ Diagrams
}

\author{
Lena-Marie Ränger *, Ulrich Preißinger and Thomas Grützner \\ Institute of Chemical Engineering, Ulm University, 89081 Ulm, Germany; ulrich.preissinger@uni-ulm.de (U.P.); \\ thomas.gruetzner@uni-ulm.de (T.G.) \\ * Correspondence: lena.raenger@uni-ulm.de
}

Received: 3 May 2018; Accepted: 28 May 2018; Published: 4 June 2018

\begin{abstract}
Dividing Wall Columns (DWCs) allow the separation of a ternary mixture in one column shell by applying a vertical partition wall, yielding a reduction of operational and capital costs of up to $30 \%$. Multiple DWC (mDWC), the consequent advancement of standard DWC, makes use of more than one partitioning wall, allowing the separation of quaternary or even higher mixtures in one column shell accompanied by a further reduction of energy consumption. Since no dedicated models for these columns are available in commercial process simulators, thermodynamic consistent flowsheets have to be designed and implemented. The thermally fully coupled Petlyuk arrangement is one important example. However, the initial convergence of these substituting flowsheets is demanding, since a large number of meaningful initial guesses have to be provided. A promising method for generating these first estimates are minimum vapor $\left(V_{\min }\right)$ diagrams. All internal flows can be extracted from these diagrams and used for robust initialization of the simulation. The goal of this work is to present the $V_{\min }$ method in a comprehensive way in order to initialize mDWC simulations to predict the separation of four component mixtures. Additionally, the adaptation of the diagram to configurations different than Petlyuk arrangements for mDWC is evaluated and a systematic procedure to obtain them is presented. In the end, an example of a converging simulation is given, which was obtained with the values from the $V_{\min }$ diagram.
\end{abstract}

Keywords: $V_{\min }$ diagram; dividing wall column; multiple dividing wall column; rigorous simulation; initialization

\section{Introduction}

Distillation captures a large amount of energy consumption in chemical processes. A typical example of an intensified process offering the opportunity to reduce this energy demand are Dividing Wall Columns (DWCs). Conventionally, the separation of a ternary mixture via distillation is performed in a sequence of two distillation columns, each having its own reboiler and condenser. To reduce the investment costs and space, the process can be combined in one column shell with a side draw for the intermediate boiling component. Nevertheless, this does not lead to a pure side product since the feed remixes with the product stream. This can be avoided by using a dividing wall in the middle of the column, which prevents backmixing, resulting in a reduction of operational costs [1-3]. Extending this method results in multiple Dividing Wall Columns (mDWC) which are able to separate multicomponent mixtures in one column shell [4]. This work focuses on mDWC for the separation of a four component feed mixture. Figure 1a shows a typical four product Dividing Wall Column with three dividing walls. 


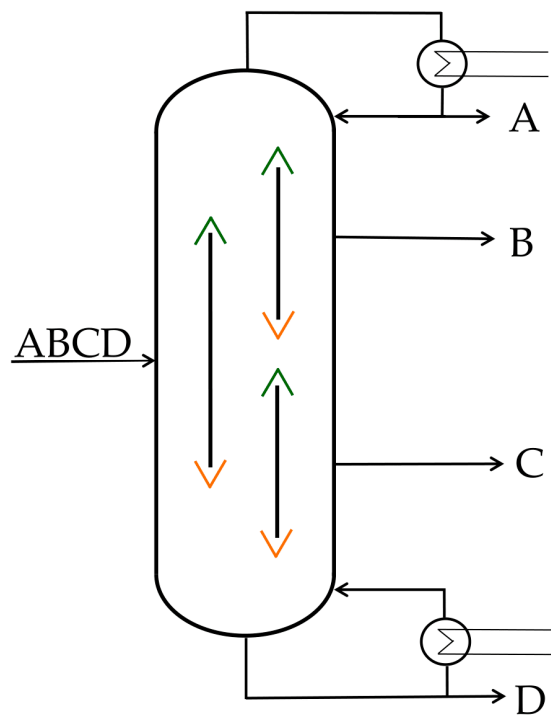

(a)

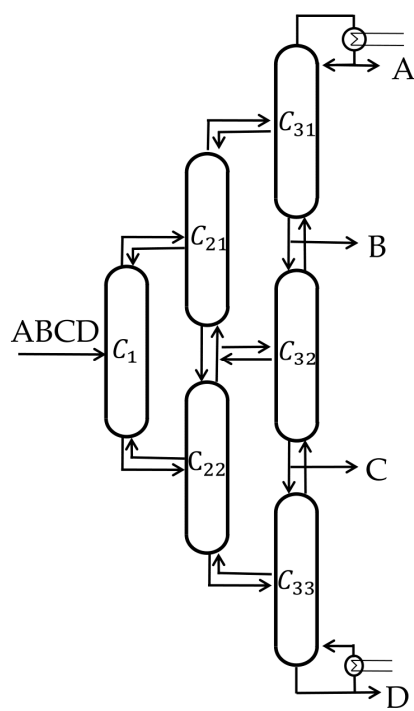

(b)

Figure 1. (a) Four product Dividing Wall Column with three dividing walls. Green: Liquid splits; Orange: Vapor splits; (b) corresponding Petlyuk sequence.

The intensification results in a higher number of degrees of freedom for mDWC compared to the conventional separation, including liquid and vapor splits at each dividing wall, as indicated as green and orange lines in Figure 1a. The liquid and vapor splits distribute the internal streams in a way that enables ideal product splits in each column section. This results in a high complexity of mDWCs, which is the reason why none have been built yet.

For the design of $\mathrm{mDWC}$, computer-based rigorous simulations are indispensable [5]. Even though there are no default models in process simulators available for Dividing Wall Columns with more than one dividing wall, there are methods to implement these that result in a thermodynamically consistent flowsheet. Among other things [2], different sections of the column can be implemented as a sequence of fully thermally coupled columns, also called the Petlyuk sequence [6]. Fully thermally coupled columns are interconnected via vapor and liquid streams. In the case of a Petlyuk sequence, only one reboiler and one condenser is present, as shown in Figure 1b. The shown Petlyuk sequence is thermally equivalent to the mDWC in Figure 1a. Note that the first column in the sequence $C_{1}$ will be called the prefractionator in the following text. In order to reduce the complexity, the number and configuration of the dividing walls can be changed. This results in equivalent thermally coupled column sequences different to the Petlyuk sequence. An example of a four product DWC with a reduced number of splits is the Kaibel column [7], which will be presented later in Section 4.2. The corresponding column sequence consists of only three thermodynamically coupled columns compared to six for the conventional Petlyuk sequence.

The main challenge of the flowsheet simulation is to obtain meaningful initial estimations of coupling streams, since these are important when attempting to reach a converging simulation. A promising method to determine initial streams for a rigorous simulation is the $V_{\min }$ method [1] presented by Halvorsen and Skogestad [8-10] based on Underwood's method [11]. $V_{\min }$ diagrams are obtained for only one column depicting different split cases. Nevertheless, they can also be applied for sequences of fully thermally coupled columns. Based on this, internal streams can be extracted and applied for the simulation initialization. In the literature, $V_{\min }$ diagrams are often only used to compare the minimum energy demand of different column sequences. In contrast to that, the $V_{\min }$ diagrams will be presented as an effective and robust tool to initialize rigorous simulations for the design of multiple Dividing Wall Columns. 
This work comprehensively summarizes the publications of Halvorsen and Skogestadt [8-10] and Fidkowski and Agrawal [12], with a focus on mDWCs. Example calculations developed by the author of this paper shall help advance understanding of the method. The basic principles have to be comprehended in order to understand the main part of this work. These are changes of $V_{\min }$ diagrams caused by changes of thermal coupling in the column sequence. A publication of Ge et al. [13] also deals with this topic. However, only the final equations are presented without a detailed description of the calculation procedure. Hence, in this work, a systematic procedure is presented which can be performed to obtain $V_{\min }$ diagrams for differently thermally coupled columns. In the end, a $V_{\min }$ diagram will be applied to a practical example to initialize a rigorous simulation.

\section{2. $V_{\min }$ Diagrams: Fundamentals}

In a conventional distillation column, there are two degrees of freedom. This means that a plot of the feed-related vapor stream $V / F$ over the feed-related distillate stream $D / F$ results in a total description of the process. Since any combination of these streams is possible, it is more meaningful to determine the minimum vapor flow $V_{\min }$ that is necessary to obtain a certain product purity. For determining $V_{\text {min }}$, Underwoods method can be used [11], for which only the feed stream, its composition, and its liquid fraction have to be known. The resulting minimum vapor flow and the corresponding distillate stream are shown in the $V_{\min }$ diagram.

An example of a ternary mixture containing components $\mathrm{ABC}$ with $\mathrm{A}$, the light boiler, and $\mathrm{C}$, the heavy boiler, is given in Figure 2. The $V_{\min }$ diagram is obtained by the concept of a simple standard distillation column with an infinite number of stages. The recovery $r$ describes the molar flow of a component in the distillate stream compared to the feed stream (see Equation (1)), having a value from zero to one. The corresponding recovery at the bottom of the column is calculated with the bottom stream.

$$
r_{i}^{T}=\frac{D_{i}}{F_{i}}
$$

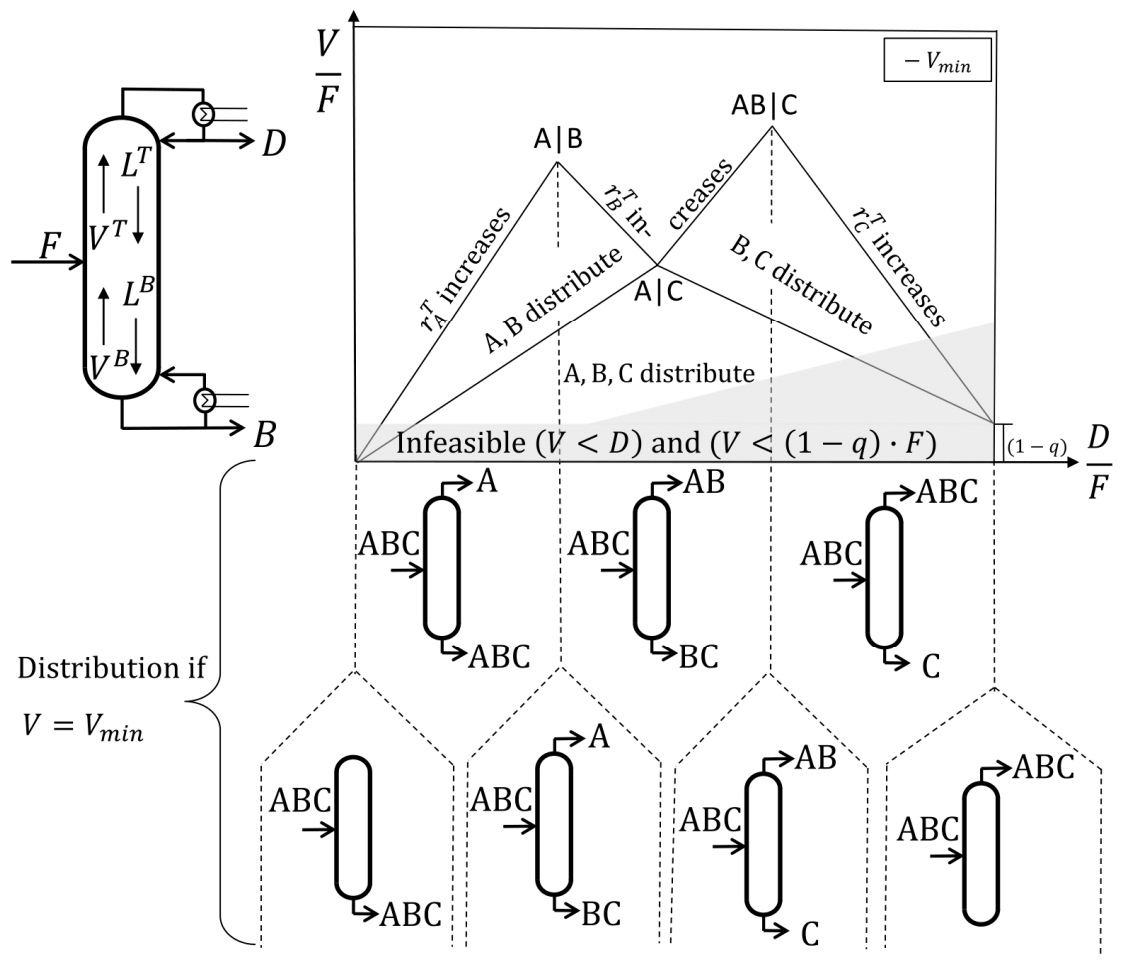

Figure 2. $V_{\min }$ diagram for a ternary mixture, where component distributions in each region are shown for the case $V=V_{\min }$. 
The internal liquid flow $L$ and vapor flow $V$ are differentiated according to their location in the column, and the superscripts $T$ and $B$ indicate the corresponding recovery in the top (distillate) and the bottom stream of the column, respectively. This syntax was adopted from the publications of Halvorsen and Skogestad [8-10], who developed the method.

In any case, there are two infeasible regions indicated by the grey-shaded area in Figure 1. First, the vapor flow inside the column cannot be smaller than the amount of vapor in the feed stream, resulting in $V<(1-q)$. F with $q$, the liquid fraction in the feed stream, to be infeasible. Second, there cannot be a higher distillate stream than vapor going up, which means that $V<D$ is infeasible. Having this in mind, the $V_{\min }$ line will be explained in the following. For a column without distillate stream, the minimum vapor flow would also be zero, and due to this, the diagram starts at $(0 \mid 0)$. Increasing the distillate stream means increasing the recovery of the light boiler, which is component $A$ in the top of the column. In order to get pure component $A$ in the distillate without impurities, the minimum vapor flow shown in the diagram as a solid line is necessary. If less vapor is used, component $B$ will also be in the distillate stream, and one says it distributes between the distillate and the bottom stream. Using more than the minimum vapor flow in a region above the $V_{\min }$ line is a waste of energy since the same product composition is reached even though more vapor is produced. Following the $V_{\min }$ line from the starting point $(0 \mid 0)$, a straight line will be found on which the recovery of component $A$ in the top of the column increases from zero to one. Reaching the recovery of one while the recoveries of the middle and heavy boiling components $B$ and $C$ are still zero is called a sharp split between $A$ and $\mathrm{B}(\mathrm{A} \mid \mathrm{B})$; in the following, it is written as the $\mathrm{AB}$ split. Sharp splits without a distributing component can be seen as maxima in the $V_{\min }$ diagram. Proceeding to higher $D / F$ ratios also causes component $\mathrm{B}$ to be found in the distillate stream in addition to $A$, resulting in a decreasing minimum vapor flow. While the recovery of component $B$ in the top of the column increases from zero to one, a minimum is passed. At that point, a sharp AIC, later denoted as AC, split at the minimum vapor demand is performed with component B distributing. For the ternary system, this split is called the preferred split of the prefractionator. A column works at the preferred split if it separates the lightest and the heaviest boiling components entering its feed stream at the corresponding minimum vapor flow. A further increase of the distillate stream after the AC split requires a higher minimum vapor flow for a distillate stream containing only component $\mathrm{A}$ and $\mathrm{B}$. This means that the $V_{\min }$ line increases until the next maximum, the sharp $\mathrm{AB} \mid \mathrm{C}$ split, is reached. Note that this split will result in the following be denoted as the $\mathrm{BC}$ split, even though component $\mathrm{A}$ is also considered to be in the top of the column. A further increase of the distillate streams means that also component $C$ will move to the top product. This results in a decrease of the vapor flow demand. Nevertheless, this region is not meaningful for separation processes.

In summary:

- There are maxima and minima describing the vapor demand for sharp splits between the components

- For a multicomponent feed mixture ( $n=$ number of components) there are generally $\frac{n \cdot(n-1)}{2}$ sharp splits, thereof $(n-1)$ without distributing component, $(n-2)$ with one distributing component etc. and $\frac{n \cdot(n-1)}{2}+2$ significant points including:

First boundary condition: no vapor results in no distillate $(0 \mid 0)$

Second boundary condition: distillate is at least the vapor fraction of the feed $(1 \mid 1-q)$

- Infeasible region: $V<D$ and $V<(1-q) \cdot F$

- Preferred split: Split between the lightest and heaviest component fed at the corresponding minimum vapor flow 


\section{Calculation of $V_{\min }$ Diagrams}

$V_{\min }$ diagrams can be calculated by an analytical solution of the Underwood equations, which will be described in greater detail in the following chapter. First, the Underwood Roots will be briefly introduced in Section 3.1 and afterwards the analytical calculation is presented in Section 3.2. In the end, the significant steps used to calculate a $V_{\min }$ diagram of a four component mixture will be shown in Section 3.3.

\subsection{Definition of Underwood Roots}

The Underwood Roots are based on a mass balance around a simple distillation column with a feed stream at its middle, with the rectifying section above the feed stage and the stripping section below. An infinite number of stages, a constant molar flow, and constant relative volatilities of the components are assumed. The latter one is denoted as $\alpha_{i}$, which is the distribution ratio of component $i K_{i}$ compared to the distribution ratio of the overall heavy boiling component $K_{H B}$ in the original feed mixture, as shown in Equation (2).

$$
\alpha_{i}=\frac{y_{i} / x_{i}}{y_{H B} / x_{H B}}=\frac{K_{i}}{K_{H B}}
$$

where $x_{i}$, is the molar fraction of component $i$ in the liquid phase and $y_{i}$, is the molar fraction of component $i$ in the vapor phase. With these assumptions, Underwood [11] defined Equations (3) and (4) [14].

$$
\begin{gathered}
\sum_{i}^{n} \frac{\alpha_{i} \cdot x_{i}^{T}}{\alpha_{i}-\Phi}=R^{T}+1 \\
\sum_{i}^{n} \frac{\alpha_{i} \cdot x_{i}^{B}}{\alpha_{i}-\Psi}=R^{B}
\end{gathered}
$$

The superscript $T$ indicates the top of the column and $B$ is the bottom; accordingly, $R^{T}$ is the reflux ratio, $R^{B}$ is the boil up ratio, and $\Phi$ and $\Psi$ are ther Underwood Roots. There are different roots above and below the feed stage, which are called actual roots:

- $\Phi$ is the actual root in the rectifying section

- $\Psi$ is the actual root in the stripping section

A pinch point analysis results in the following definitions for the actual roots: Equation (5) for the rectifying section and (6) for the stripping section [14].

$$
\begin{gathered}
\Phi=\frac{L^{T}}{V^{T} \cdot K_{H B}} \\
\Psi=\frac{L^{B}}{V^{B} \cdot K_{H B}}
\end{gathered}
$$

$L$ is the molar liquid stream and $V$ the molar vapor stream. These internal streams differ depending on the performed product split. Hence, there are as many actual roots as components $n$ in each section and their values are between the values of the relative volatilities of each component $\alpha_{i}$ (Equation (7)).

$$
\begin{aligned}
& \alpha_{1}>\Phi_{1}>\alpha_{2}>\Phi_{2}>\ldots>\Phi_{n} \\
& \Psi_{1}>\alpha_{1}>\Psi_{2}>\alpha_{2}>\ldots>\alpha_{n}
\end{aligned}
$$

A decreasing vapor flow results in increasing $\Psi$ and decreasing $\Phi$, which means that there is an intersection of the top and bottom roots. At this value, the minimum vapor flow $V_{\min }$ is reached and the roots are called common roots $\theta$. There are $n-1$ common roots fulfilling Equation (8).

$$
\alpha_{1}>\Phi_{1}=\Psi_{2}=\theta_{1}>\alpha_{2} \ldots>\alpha_{n}
$$


The common roots are called active roots $\theta^{*}$ if their value lies between the values of relative volatilities of the components, which are split as shown in Figure 3a. With $n_{\text {dist }}$, the number of distributing components (components with $r_{i}^{T} \neq 1$ or 0 ) there is $n_{\text {dist }}+1$ active roots:

- For sharp splits (no distributing component), there is always only one active root, which is the one with a value between the volatilities of the components split

- For sharp splits with one distributing component, there are two active roots

- For sharp splits with two distributing components, there are three active roots etc.

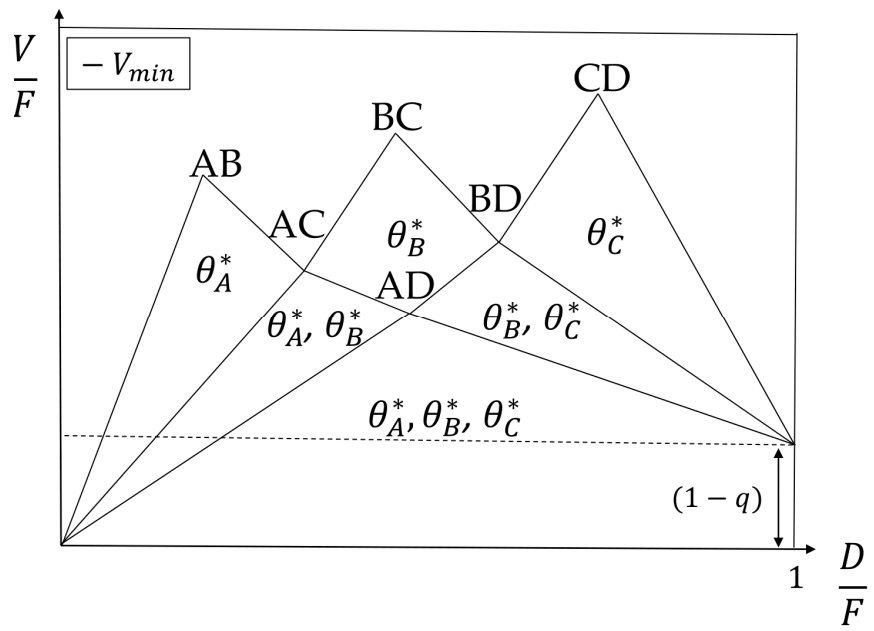

(a)

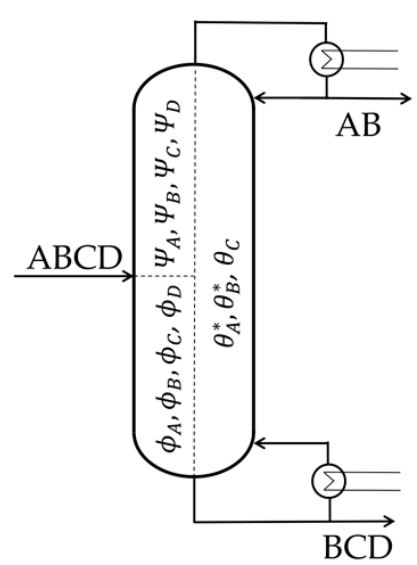

(b)

Figure 3. (a) Active roots during different splits shown in $V_{\min }$ diagram of a four component mixture (modified after [10]) and (b) different kinds of Underwood Roots in a distillation column for a sharp AC split and four components fed.

Figure 3b symbolically shows all kinds of Underwood Roots in a distillation column for a sharp AC split with four components fed.

\subsection{Analytical Calculation of $V_{\min }$ Diagrams}

$V_{\min }$ diagrams are based on the Underwood Roots. The general calculation procedure is always the same, independent of the number of components. First, the Underwood Roots of the feed stream have to be calculated (Section 3.2.1). Second, in the case of sharp splits with distributing components, the recoveries of the distributing components have to be determined. If there are no splits with distributing components, this step is canceled. Third, the minimum vapor flow, as well as the distillate streams, can be calculated with the active roots and the recoveries (step 2 and 3 are presented in Section 3.2.2). Note that only the extreme points of the $V_{\min }$ diagram are calculated, which can be connected by straight lines. For a better understanding, the procedure is explained for a four component system in Section 3.2.3.

\subsubsection{Calculation of the Common Roots}

The common roots of the feed mixture are calculated with the first Underwood equation which is also called the feed equation (Equation (12)). In the following, the derivation of the equation is presented. First, a vapor flow balance around the column is performed, as shown in Figure 4. 


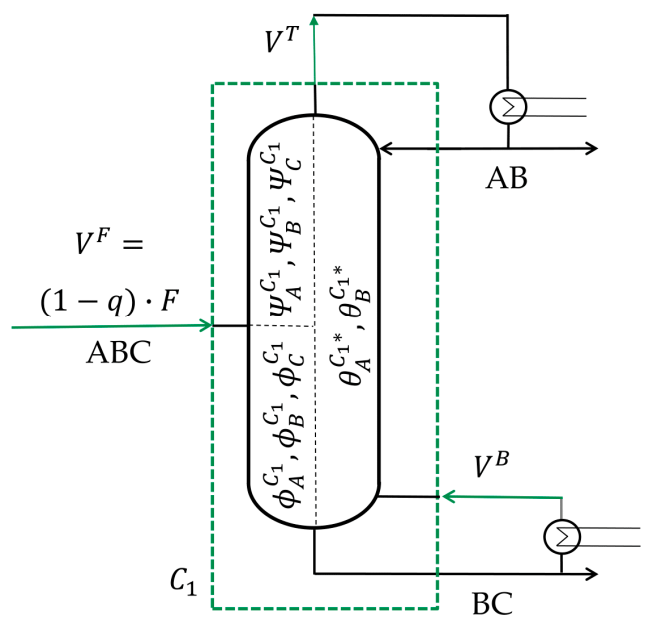

Figure 4. Vapor balance around a column $C_{1}$ for the separation of a three component mixture ABC.

Where $F$ is the feed stream and $V$ is the vapor stream; the superscripts $B, T$, and $F$ indicate the streams at the bottom and top of the column and the feed stream, respectively; and $q$ is the liquid fraction of the feed. The vapor balance results in Equation (9).

$$
(1-q) \cdot F+V^{B}=V^{T}
$$

Based on the Underwood Roots, the vapor stream in the top of the column is defined by Equation (10) (for the derivation, see [15]).

$$
V^{T}=\sum_{i=1}^{n} \frac{\alpha_{i} \cdot D_{i}}{\alpha_{i}-\Phi}=\sum_{i=1}^{n} \frac{\alpha_{i} \cdot F \cdot z_{i} \cdot r_{i}^{T}}{\alpha_{i}-\Phi}
$$

$D_{i}$ is the molar flow of component $i$ in the distillate stream, $z_{i}$ is the molar concentration of component $i$ in the feed stream, and $r_{i}^{T}$ is the recovery of the component in the distillate stream (Equation (1)). The vapor flow in the bottom of the column is defined by Equation (11).

$$
V^{B}=\sum_{i=1}^{n} \frac{\alpha_{i} \cdot B_{i}}{\alpha_{i}-\Psi}=\sum_{i=1}^{n} \frac{\alpha_{i} \cdot F \cdot z_{i} \cdot r_{i}^{B}}{\alpha_{i}-\Psi}
$$

$B_{i}$ is the molar flow of component $i$ in the bottom stream and $r_{i}^{B}$ is the corresponding recovery related to the fed stream of the component. The minimum vapor flow is reached at $\Phi=\Psi=\theta$, with $r_{i}^{B}+r_{i}^{T}=1$. A combination of Equations (9)-(11) results in the so called feed equation, which is used to calculate the common roots (Equation (12)). Note that $r_{i}^{B}$ is defined for the outgoing bottom product, whereas the bottom vapor stream is incoming, which results in a negative sign that causes the recoveries to be canceled out.

$$
V^{F}=(1-q) \cdot F=\sum_{i=1}^{n} \frac{\alpha_{i} \cdot F \cdot z_{i}}{\alpha_{i}-\theta}
$$

\subsubsection{Calculation of Minimum Vapor Flow}

The second Underwood equation (Equation (13)) is used to calculate $V_{\min }$ depending on the active $\operatorname{root} \theta^{*}$. Note that Equation (13) represents Equation (10) for the $V_{\min }$ case, meaning $\Phi=\theta$.

$$
V_{\min }=\sum_{i=1}^{n} \frac{\alpha_{i} \cdot D_{i}}{\alpha_{i}-\theta^{*}}=\sum_{i=1}^{n} \frac{\alpha_{i} \cdot z_{i} \cdot F}{\alpha_{i}-\theta^{*}} \cdot r_{i}^{T}
$$


The index * means that only the active root of the split is used for the calculation. The corresponding distillate stream is calculated according to Equation (14).

$$
D=\sum_{i=1}^{n} z_{i} \cdot r_{i}^{T} \cdot F
$$

The obtained data for $V_{\min }$ and $D$ divided by the feed stream are plotted in the $V_{\min }$ diagram.

\subsubsection{Example: $V_{\min }$ Diagram of a Four Component Feed}

The relative volatilities of the feed components can be approximated manually by the extended Raoult's Law, which is also implemented in simulation programs as Aspen HYSIS ${ }^{\circledR}$. There, the K-values of each component in the mixture can be read out and the relative volatilities $\alpha_{i}$ are determined with Equation (2). The common roots are calculated with Equation (12). Applying Equation (13) results in three equations that are needed to calculate the $V_{\min }$ diagram: Equation (15) for the first root $\mathrm{A}$, Equation (16) for root B, and Equation (17) for root C.

$$
\begin{aligned}
& \frac{V_{\min }}{F}=\frac{\alpha_{A} \cdot z_{A}}{\alpha_{A}-\theta_{A}^{*}} \cdot r_{A}^{T}+\frac{\alpha_{B} \cdot z_{B}}{\alpha_{B}-\theta_{A}^{*}} \cdot r_{B}^{T}+\frac{\alpha_{C} \cdot z_{C}}{\alpha_{C}-\theta_{A}^{*}} \cdot r_{C}^{T}+\frac{\alpha_{D} \cdot z_{D}}{\alpha_{D}-\theta_{A}^{*}} \cdot r_{D}^{T} \\
& \frac{V_{\min }}{F}=\frac{\alpha_{A} \cdot z_{A}}{\alpha_{A}-\theta_{B}^{*}} \cdot r_{A}^{T}+\frac{\alpha_{B} \cdot z_{B}}{\alpha_{B}-\theta_{B}^{*}} \cdot r_{B}^{T}+\frac{\alpha_{C} \cdot z_{C}}{\alpha_{C}-\theta_{B}^{*}} \cdot r_{C}^{T}+\frac{\alpha_{D} \cdot z_{D}}{\alpha_{D}-\theta_{B}^{*}} \cdot r_{D}^{T} \\
& \frac{V_{\min }}{F}=\frac{\alpha_{A} \cdot z_{A}}{\alpha_{A}-\theta_{C}^{*}} \cdot r_{A}^{T}+\frac{\alpha_{B} \cdot z_{B}}{\alpha_{B}-\theta_{C}^{*}} \cdot r_{B}^{T}+\frac{\alpha_{C} \cdot z_{C}}{\alpha_{C}-\theta_{C}^{*}} \cdot r_{C}^{T}+\frac{\alpha_{D} \cdot z_{D}}{\alpha_{D}-\theta_{C}^{*}} \cdot r_{D}^{T}
\end{aligned}
$$

The terms $\frac{\alpha_{i} \cdot z_{i}}{\alpha_{i}-\theta_{i}^{*}}$ from Equations (15) to (17) are known, whereas the unknowns are the recoveries and $V_{\min }$. Depending on the splits, there are different combinations for the recoveries. The combinations are shown in Table 1. Component A is always in the top stream of the column $\left(r_{A}^{T}=1\right)$ and $\mathrm{D}$ is always

\begin{tabular}{|c|c|c|c|c|c|c|}
\hline $\begin{array}{ll} & \text { Splits } \\
\text { Comp. } & \end{array}$ & AB & AC & BC & $\mathrm{AD}$ & BD & CD \\
\hline$r_{A}^{T}$ & 1 & 1 & 1 & 1 & 1 & 1 \\
\hline$r_{B}^{A}$ & 0 & Unknown & 1 & Unknown & 1 & 1 \\
\hline$r_{C}^{B}$ & 0 & 0 & 0 & Unknown & Unknown & 1 \\
\hline$r_{D}^{T}$ & 0 & 0 & 0 & 0 & 0 & 0 \\
\hline
\end{tabular}
in the bottom stream $\left(r_{D}^{T}=0\right)$, so the last term in Equations (15) to (17) can be canceled.

Table 1. Recovery matrix describing different splits for the separation of a four components mixture containing ABCD.

If, for example, components B and C are separated in a sharp split, component A is completely in the top of the column, as well as component $\mathrm{B}$, whereas component $\mathrm{C}$ and $\mathrm{D}$ are at the bottom. If there are distributing components, their recovery in the top of the column for a sharp split is unknown. The unknown recoveries, as well as the corresponding minimum vapor flows for the splits, are calculated with Equations (15)-(17). Which of these equations has to be applied is given by the active roots, which depend on the performed split, as shown in Table 2 and Figure 3a.

Table 2. Active roots (indicated by *) during different splits for separation of four components ABCD (from Figure 3a).

\begin{tabular}{ccccccc}
\hline Splits & AB & AC & BC & AD & BD & CD \\
\hline$\theta_{A}$ (Equation (15)) & $*$ & $*$ & & $*$ & & \\
$\theta_{B}$ (Equation (16)) & & $*$ & $*$ & $*$ & $*$ & \\
$\theta_{C}$ (Equation (17)) & & & & $*$ & $*$ & $*$ \\
\hline
\end{tabular}


With the knowledge of the active roots for the splits, the $V_{\min }$ diagram can be calculated as follows [12]:

- Sharp splits: One unknown and one equation
AB: $V_{\min }$ unknown, active $\operatorname{root} \theta_{A}{ }^{*}$, Equation (15)
○ BC: $V_{\min }$ unknown, active $\operatorname{root} \theta_{B}{ }^{*}$, Equation (16)
$\bigcirc \quad \mathrm{CD}: V_{\min }$ unknown, active $\operatorname{root} \theta_{C}^{*}$, Equation (17)

- Sharp splits with one distributing component: Two unknowns and two equations
$\bigcirc \quad$ AC: $V_{\min }$ and $r_{B}^{T}$ unknown, active $\operatorname{root} \theta_{A}{ }^{*}$ and $\theta_{B}{ }^{*}$, Equations (15) and (16)
$\bigcirc \quad$ BD: $V_{\min }$ and $r_{C}^{T}$ unknown, active root $\theta_{B}{ }^{*}$ and $\theta_{C}{ }^{*}$, Equations (16) and (17)

- Sharp splits with two distributing components: Three unknowns and three equations

$\bigcirc \quad \mathrm{AD}: V_{\min } ; r_{B}^{T}$ and $r_{C}^{T}$ unknown; active roots $\theta_{A}{ }^{*}, \theta_{B}{ }^{*}$ and $\theta_{C}{ }^{*}$, Equations (15)-(17)

- Afterwards, the corresponding distillate stream $D$ of each $V_{\min }$ can be calculated with Equation (14)

This calculation procedure can for example be implemented in MATLAB, a commercial computational program. Then, only the feed stream properties have to be specified and the whole $V_{\min }$ diagram is calculated. Based on this code, $V_{\min }$ diagrams for different mixtures can be obtained with very low effort.

\section{3. $V_{\min }$ Diagrams by Rigorous Simulations}

$V_{\min }$ diagrams can also be obtained by using a rigorous commercial process simulator such as Aspen Plus ${ }^{\circledR}$. For this purpose, only one column is needed, as shown in Figure 2 (e.g., RadFrac). Design specifications are used to describe the splits. This assumes a three component feed consisting of components A, B, and C. The minimum vapor flow for the sharp AB split shall be calculated, and in order to do this, a design specification is added as local specification for the block. The design specification in this case is a very small mole fraction of component $\mathrm{A}$ in the bottom stream (e.g., 0.0001), which is the same for the mole fraction of component B in the top stream. If a sharp split with distributing components as $\mathrm{AC}$ is calculated, the mole purity of component $\mathrm{A}$ in the bottom is still set to a very small value, such as 0.0001 , whereas now the mole fraction of component $C$ in the top is also set to this small value. This procedure is easily extended to multicomponent mixtures. The vapor flow directly results from the simulation. Since a detailed description of the procedure is given in the literature [8], this will not be discussed further.

\section{4. $V_{\min }$ Diagrams for Specific Column Configurations}

For fully thermally coupled columns, the actual Underwood Roots of the feed mixture entering a column carry over to subsequent columns. This means that the actual root in the top of a column $\Phi_{i}$ carries over with the distillate stream to the following column, where it becomes the common root. The same occurs for the bottom stream, where the actual roots in the bottom of the column $\Psi_{i}$ carry over with the bottom stream to the subsequent column and become the common root for the following split [16]. Note that, formally, there is no longer a distillate and a bottom stream for fully thermally coupled columns. Nevertheless, the distillate stream is plotted in the $V_{\min }$ diagram. For the thermally coupled columns, they are a kind of pseudo stream. The distillate stream describes the difference in vapor stream leaving and liquid stream entering at the top of the column, respectively, for the bottom stream. In order to keep the syntax of the original $V_{\min }$ diagram of a single column the same, they are still specified as these. 


\subsection{Petlyuk Cofiguration}

In the Petlyuk configuration, each column splits the heaviest and lightest boiling components entering the column at the corresponding minimum vapor flow. This also means that the intermediate boiling components are distributed between the top and the bottom stream. It is said that the columns work at their preferred splits. For each column working at its preferred split, the actual Underwood Roots that carry over are also the common roots from the pre-sequent column. This means that the $V_{\min }$ diagram of a feed mixture in a conventional column is also valid for a Petlyuk configuration without changes. Note that the common roots $\theta_{i}$ can only carry over if they are active. Figure 5a shows a Petlyuk configuration and Figure $5 \mathrm{~b}$ the corresponding $V_{\min }$ diagram.

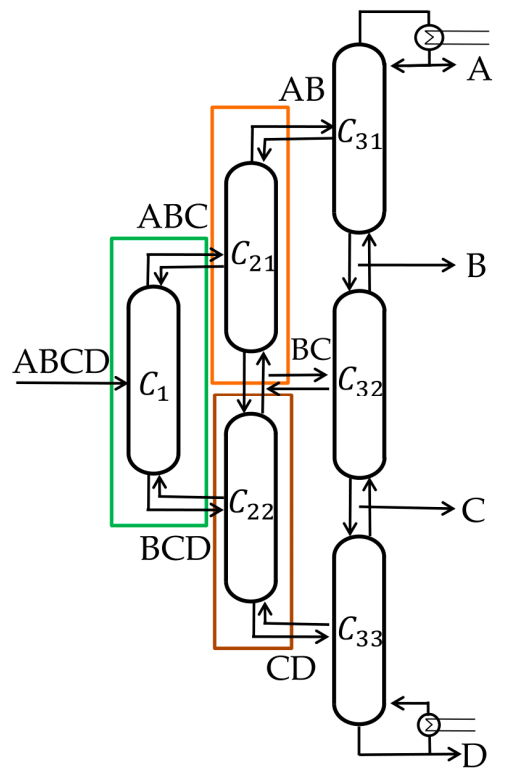

(a)

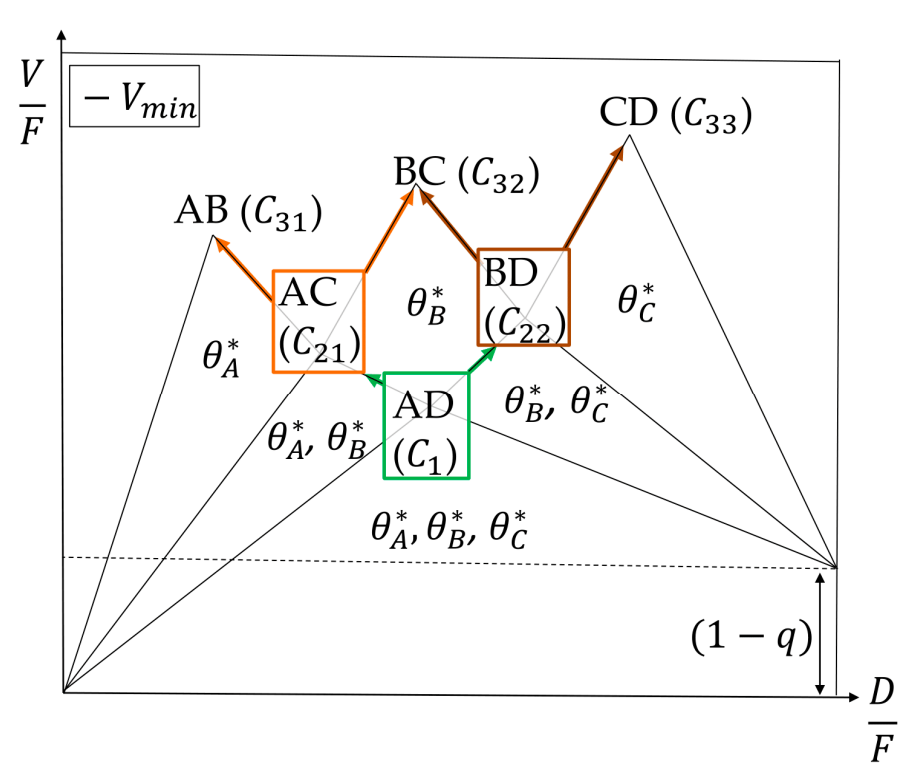

(b)

Figure 5. (a) Petlyuk configuration for a four component split and (b) corresponding $V_{\text {min }}$ diagram showing the carryover of common Underwood Roots (modified after [10]).

The first column (prefractionator, $C_{1}$ ) splits component $A$ and component $\mathrm{D}$ at the corresponding minimum vapor flow (its preferred split). This split is the lowest $V_{\min }$ point in the diagram. In this case, the common roots $\mathrm{A}, \mathrm{B}$, and $\mathrm{C}$ are active. In the column that is connected to the distillate stream of the prefractionator, $C_{21}$, component $A$ is the lightest and $C$ the heaviest boiling component which are split (again preferred split). The common roots $\mathrm{A}$ and B, lying between the volatilities of component $\mathrm{A}$ and $\mathrm{C}$, carry over to this column. The same is true for the column connected to the bottom stream of the prefractionator $\left(C_{22}\right)$. Component $\mathrm{B}$ is the lightest and $\mathrm{D}$ the heaviest boiling component. The roots $\mathrm{B}$ and $C$ carry over and describe the split between the two components. The same is true for the following sharp splits without distributing components. As long as each thermally coupled column is allowed to work at its preferred split, the $V_{\min }$ diagram will not change. Note that the highest peak in the diagram shows the total necessary vapor needed to separate the feed mixture in a Petlyuk configuration.

With the $V_{\min }$ diagram, all coupling streams in a Petlyuk configuration, meaning the vapor, as well as liquid streams, can be determined as shown in Figure 6. 


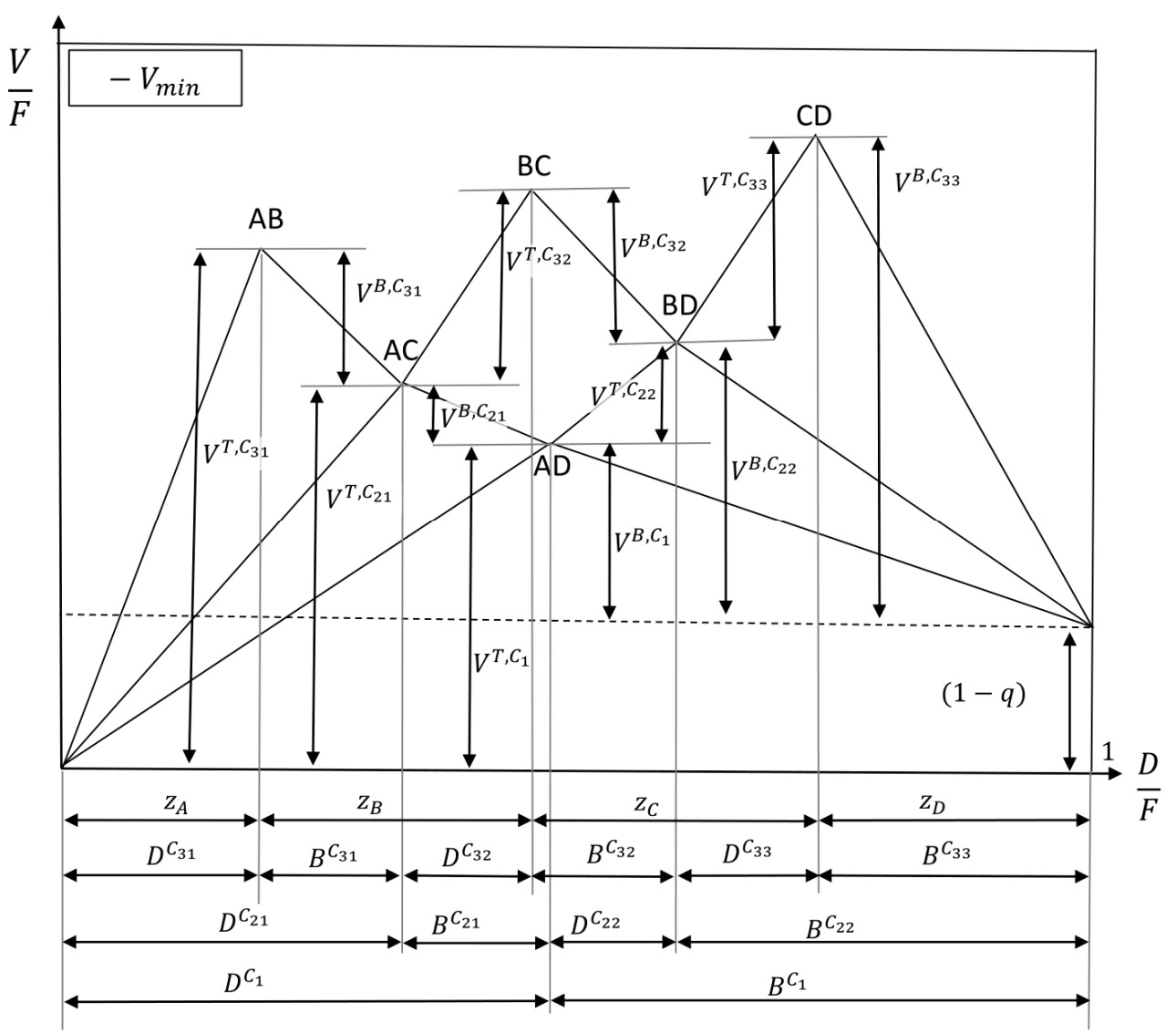

Figure 6. Extracting internal streams from the $V_{\text {min }}$ diagram for a four product Petlyuk configuration (adapted after [10]). For the column numbering, see Figure $1 b$.

The vapor streams can always be calculated by a balance around the particular columns. For example, the vapor stream at the top of column $C_{1}\left(V^{T, C_{1}}\right)$ is the vapor stream entering at the bottom $\left(V^{B, C_{1}}\right)$ plus the amount of vapor in the feed stream $(1-q)$. The column $C_{21}$ is fed with the vapor of column $C_{1}$. Therefore, the internal vapor stream at the bottom of the column $\left(V^{B, C_{21}}\right)$ is the difference between the performed split (AC) and the prior split (AD) $\left(V^{B, C_{21}}=V_{\min , A C}-V_{\min , A D}\right)$. Internal liquid streams are determined by a molar balance at the head of the column, resulting in Equation (18).

$$
L^{T, C_{i}}=V^{T, C_{i}}-D^{C_{i}}
$$

Also note the difference between the vapor at the top and the bottom of the columns, for example, in column $C_{33}$, which performs the split between component $C$ and $D$. The bottom stream is the vapor which is needed to separate $C$ and $D$, including the prior split from $A$ and $B$, which can also be written as the $A B C I D$ split. The vapor in the top stream is only the vapor needed to split components $C$ and $D$ entering the column from the prior one. The internal feed stream entering only contains component $C$ and D. This means that the top vapor stream only describes the vapor needed for the CID split.

\subsection{Kaibel Configuration}

The Kaibel column is a simplified configuration that enables the separation of four components in one column shell with only one dividing wall. It is the only yet realized DWC to separate four component mixtures $[7,17]$. The corresponding thermodynamic equivalent sequence of thermally coupled columns is shown in Figure 7a. One major difference of the Kaibel column compared to the conventional Petlyuk sequence from Figure $5 a$ is the split in the prefractionator. It is not working at 
its preferred split which is $\mathrm{AD}$, but at a sharp $\mathrm{BC}$ split. Hence, the columns are no longer working in the most energy efficient way, resulting in a higher energy consumption of the Kaibel configuration compared to the Petlyuk configuration in any case [18]. The $V_{\min }$ diagram of the original feed is still valid, but only in the prefractionator, and at the $B C$ instead of the $A D$ split. The minimum vapor flow of the subsequent columns has to be recalculated and results in a change of the $V_{\min }$ diagram, as shown in Figure $7 \mathrm{~b}$. This is discussed in the following.

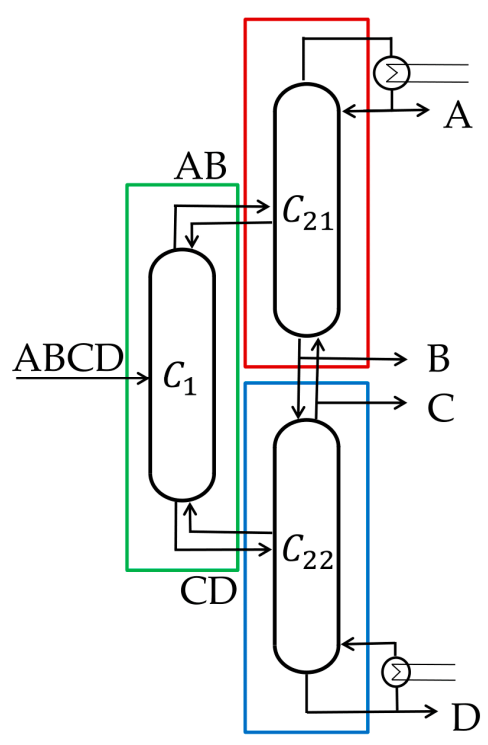

(a)

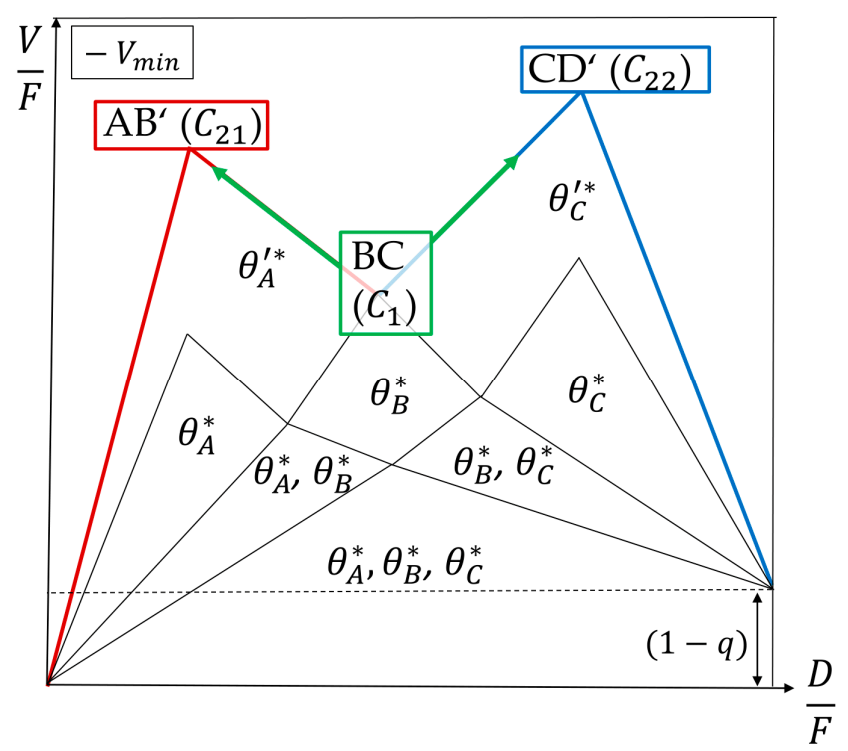

(b)

Figure 7. (a) Kaibel configuration for four component split and (b) corresponding $V_{\text {min }}$ diagram. Red lines indicate the new AB split and blue lines the new CD split (modified after [10]).

For feasible processes, the vapor flow in subsequent columns is always higher than in the pre-sequent. Accordingly, performing the column $C_{21}$ with a lower vapor stream than $C_{1}$ is impossible. Therefore, it is obvious that the $V_{\min }$ diagram must change. A closer look at the Underwood Roots also shows that the diagram has to be adapted. The only active root in $C_{1}$ is common root $\mathrm{B} \theta_{B}$ because the $B C$ split is performed, which is not the preferred one. The common roots $A$ and $C$ are not active and cannot carry over. Only the actual roots of the prefractionator carry over, which means that a performance at minimum vapor flow is no longer feasible. To determine the new $V_{\min }$ diagram for a Kaibel configuration, first the $V_{\min }$ diagram of the prefractionator $C_{1}$ is calculated, as described in Section 3.2.3. The active roots in $C_{21} \theta_{A}{ }^{\prime}$ and $C_{22} \theta_{C}{ }^{\prime}$ are calculated with a new feed equation, which is derived by a vapor balance around the corresponding column, as explained in Section 3.2.1. The resulting feed equation used to calculate $\theta_{A}{ }^{\prime}$ in $C_{21}$ is shown in Equation (19).

$$
V_{\min , B C}=V^{T, C_{21}}-V^{B, C_{21}}=\sum_{i=1}^{n} \frac{\alpha_{i} \cdot z_{i} \cdot F}{\alpha_{i}-\theta_{A}^{\prime}} \cdot r_{i}^{T, C_{1}}=\frac{\alpha_{A} \cdot F \cdot z_{A} \cdot r_{A}^{T, C_{1}}}{\alpha_{A}-\theta_{A}^{\prime}}+\frac{\alpha_{B} \cdot F \cdot z_{B} \cdot r_{B}^{T, C_{1}}}{\alpha_{B}-\theta_{A}^{\prime}}
$$

$V_{\min , B C}$ is known from the $V_{\min }$ diagram which is valid in the prefractionator and the recoveries of $\mathrm{A}$ and $\mathrm{B}$ are one. The terms for component $\mathrm{C}$ and $\mathrm{D}$ were canceled out because their recoveries at column $C_{1}$ are zero. The resulting new minimum vapor flow for the $\mathrm{AB}$ split is shown in Equation (20). Due to the fact that vapor is fed to the middle of column $C_{21}$, the minimum vapor flow of the $\mathrm{AB}$ split equals the vapor flow at the top of the column (compare to Figure 6).

$$
V_{\min , A B^{\prime}}=V^{T, C_{21}}=\frac{\alpha_{A} \cdot F \cdot z_{A}}{\alpha_{A}-\theta_{A}^{\prime}}
$$


The feed equation in column $C_{22}$ is also derived by a vapor balance. Now, component $C$ and $\mathrm{D}$ are in the feed stream to column $C_{22}$ and $V_{\min , B C}$ is a leaving stream instead of an entering stream. Equation (21) is used to calculate the new common root $C\left(\theta_{C}^{\prime}\right)$.

$$
-V_{\min , B C}+(1-q) \cdot F=V^{T, C_{22}}-V^{B, C_{22}}=\sum_{i=1}^{n} \frac{\alpha_{i} \cdot z_{i} \cdot F}{\alpha_{i}-\theta_{C}^{\prime}} \cdot r_{i}^{B, C_{1}}=\frac{\alpha_{C} \cdot F \cdot z_{C} \cdot\left(1-r_{C}^{T, C_{1}}\right)}{\alpha_{C}-\theta_{C}^{\prime}}+\frac{\alpha_{D} \cdot F \cdot z_{D} \cdot\left(1-r_{D}^{T, C_{1}}\right)}{\alpha_{D}-\theta_{C}^{\prime}}
$$

It is assumed that the composition of the vapor from $C_{22}$ to $C_{1}$ is the same as the one in the liquid stream leaving $C_{1}$ at the bottom to $C_{22}$. The recoveries of $C$ and $D$ in the top stream of $C_{1}$ are zero. Now note that the new common root $\theta_{C}^{\prime}$ is only valid for the feed stream entering $C_{22}$ only containing component $C$ and $D$ and not $A$ and $B$. In addition to that, since there is vapor leaving the column at the middle, the minimum vapor for the $C D$ split equals the bottoms vapor stream and not the top vapor stream. Based on this, Equation (22) is used for the calculation of the new CD split.

$$
V_{\min , C D^{\prime}}=V^{B, C_{22}}+(1-q) \cdot F=V^{T, C_{22}}+V_{\min , B C}-(1-q) \cdot F+(1-q) \cdot F=\frac{\alpha_{C} \cdot F \cdot z_{C} \cdot r_{C}^{T, C_{22}}}{\alpha_{C}-\theta_{C}^{\prime}}+V_{\min , B C}
$$

with a recovery of component $C$ at the top of column $C_{22} r_{C}^{T, C_{22}}=1$.

Another possibility to determine the new common roots would be to calculate the actual roots in column $C_{1}$. Thus, also a vapor balance is made, but in this case, around $C_{1}$ instead of $C_{21} / C_{22}$, as shown in Equation (23) for the top section of $C_{1}$.

$$
V^{T, C_{1}}=\frac{\alpha_{A} \cdot F \cdot z_{A} \cdot r_{A}^{T, C_{1}}}{\alpha_{A}-\Phi^{C_{1}}}+\frac{\alpha_{B} \cdot F \cdot z_{B} \cdot r_{B}^{T, C_{1}}}{\alpha_{B}-\Phi^{C_{1}}}=V_{\min , B C}
$$

with $\Phi^{C_{1}}=\theta^{C_{21}}=\theta_{A}^{\prime}$, both calculations result in the same values for the common roots in $C_{21}$ and $C_{22}$.

\section{3. $V_{\min }$ Diagrams for Other Column Configurations}

As explained before (Section 4.2), the $V_{\min }$ diagram changes as soon as one column does not work at its preferred split. This means that it does not perform the sharp split between the lightest and heaviest boiling component in its feed stream at the minimum vapor flow. This results in a change of the common and therefore active roots.

Adapted column configurations can be important in order to reduce the complexity of the process, for example, by minimizing the number of vapor splits, as presented by Ge et al. [13]. An adapted column sequence results in changes of thermal coupling and thus the $V_{\min }$ diagram. In order to obtain the new $V_{\min }$ diagram in the subsequent columns, a systematic procedure is suggested, as shown in Figure 8.

As soon as one column is not working at the preferred split, some of the common roots cannot carry over to the following columns. This can be the case if a split is skipped or if more vapor is provided than the minimum vapor necessary. Both cases result in a change of common root(s). In the first case, new common root(s) are calculated by determining the feed equation for the subsequent column. This is performed with a vapor balance as shown in Section 4.2. Note that the feed stream to the subsequent columns is different than the feed to the first column; for $C_{j+1}^{T}$, the feed is the distillate stream of $C_{j}$, and for $C_{j+1}^{B}$, it is the bottom stream of $C_{j}$. In the second case, more vapor is provided than necessary $\left(V>V_{\text {min }}\right)$, which results in a new common root in the corresponding column which carries over to subsequent columns. Generally, the flowsheet in Figure 8 enables a total calculation of the $V_{\min }$ diagram based on the original feed composition, no matter which sequence of thermally coupled columns is used. 


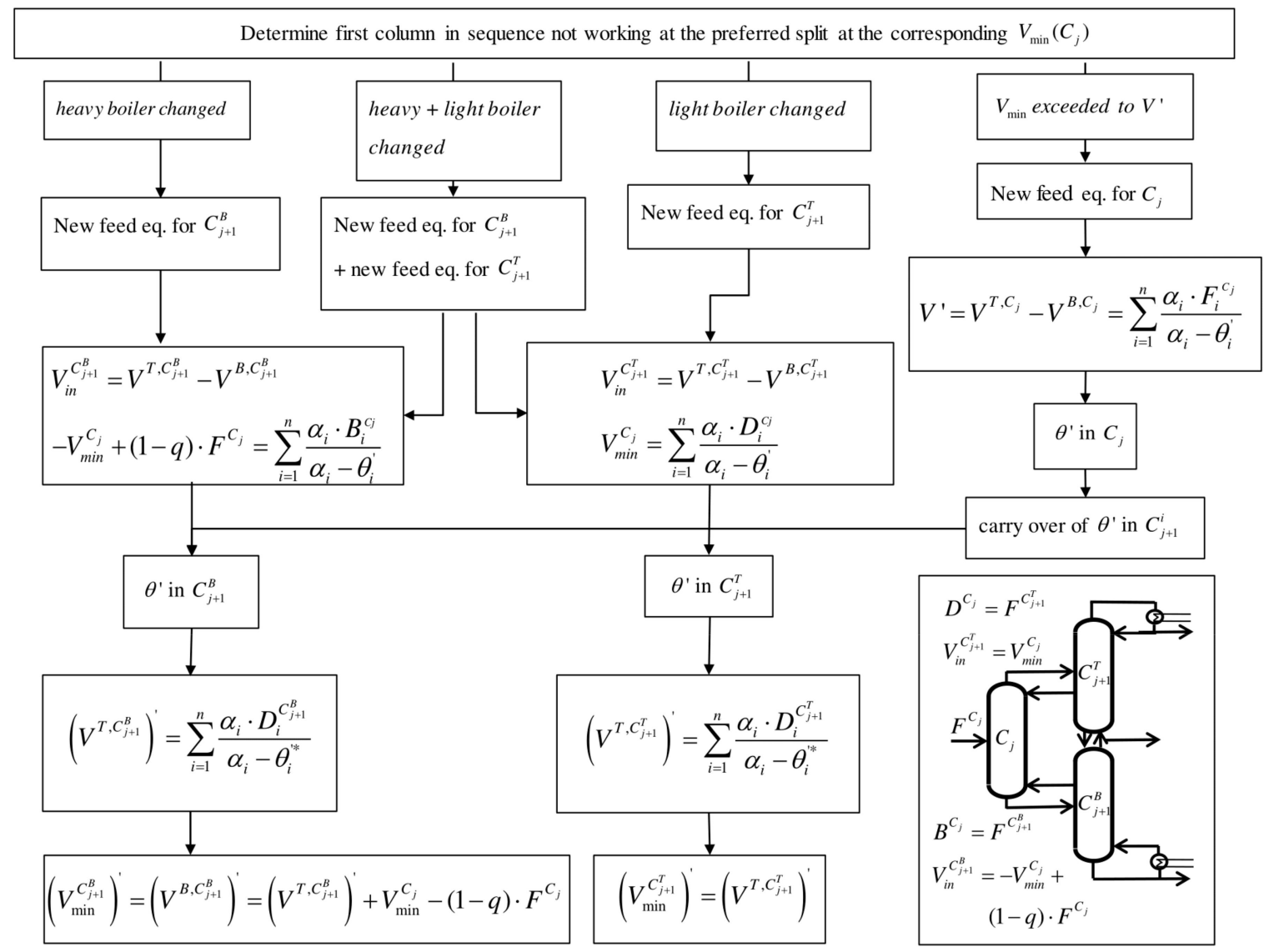

Figure 8. Schematic procedure to determine changes in the $V_{\min }$ diagram if there are columns not working at the preferred split.

\section{Initialization of Rigorous Simulation}

For the initialization of a simulation via $V_{\min }$ diagrams, one point has to be considered if liquid side streams are assumed. No vapor leaving in side streams and assuming a feed with $q=1$ means that the whole amount of vapor produced by the reboiler at the bottom of $C_{33}$ is leaving column $C_{31}$ at the top. This means that all sharp splits without distributing components are performed at the vapor amount of the most difficult split. Hence, for the case of liquid side streams, not the minimum vapor flow of each split can be implemented in the simulation [19]. There are also other methods to compensate for an excess of vapor, for example, a vapor side stream or a distribution of the vapor in the whole column arrangement instead of only in the columns in the last row. Small differences can be compensated for by using superheated or subcooled feed streams, as presented in [4]. Nevertheless, these cases will not be considered in this work.

A multiple Dividing Wall Column model for the separation of four components is presented, which was simulated in Aspen Plus ${ }^{\circledR}$. It has to be kept in mind that an optimal solution is not searched for, and the main goal is to get a simulation that converges with the initial values. The system 3-Metylhexane (A)/Toluene (B)/Ethylbenzene (C)/1-Methyl-3-Ethylbenzene (D) was used for the simulation $\left(z_{i}=0.25\right)$. First, the $V_{\min }$ diagram was calculated, which is shown in Figure 9, with a MATLAB code performing the calculation presented in 3.2.3. K-values of the feed $(q=1)$ were determined in Aspen HYSIS ${ }^{\circledR}$ and are all divided by the $K$-value of the heavy boiler 1-Methyl-3-Ethylbenzene according to Equation (2), resulting in $\alpha_{i}[7.5,4.5,2.2,1]$. 


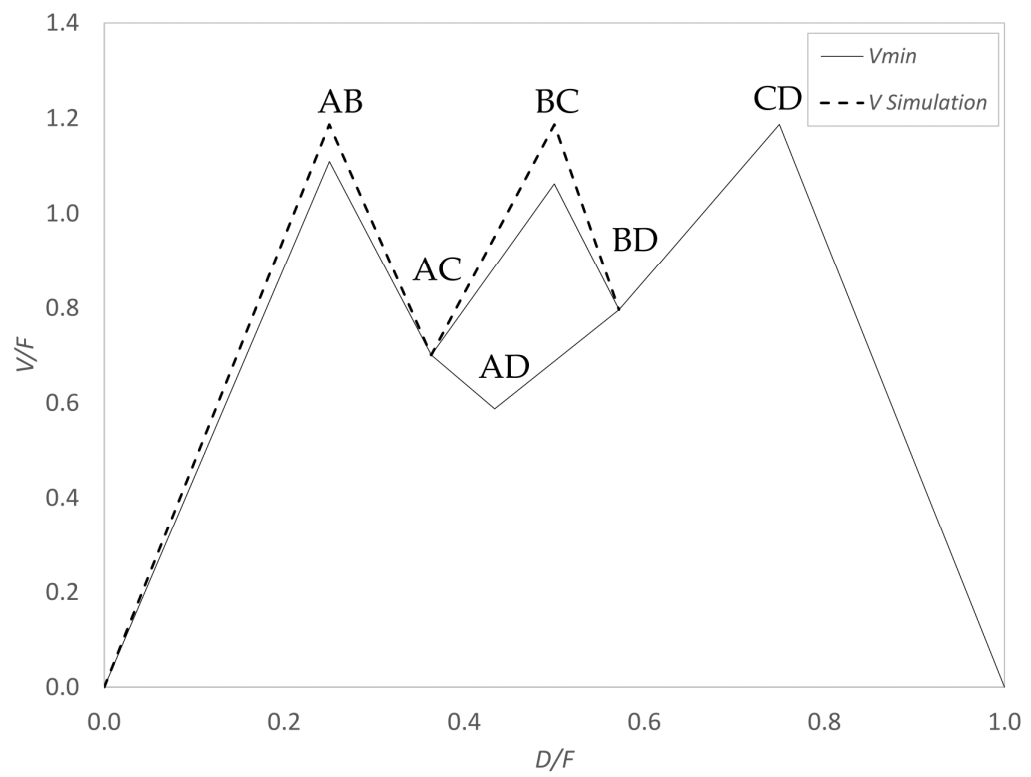

Figure 9. $V_{\min }$ diagram of quaternary mixture of 3-Metylhexane (A)/Toluene (B)/Ethylbenzene (C) /1-Methyl-3-Ethylbenzene (D) $\left(z_{i}=0.25, q=1\right)$. Dashed line indicates vapor streams implemented in simulation in Aspen Plus ${ }^{\circledR}$.

The most difficult split is the $C D$ split. As explained before, this results in the same vapor amount at the $\mathrm{AB}$ as well as $\mathrm{BC}$ peak for the simulation, which is indicated as a dashed line in the diagram. Internal streams were read out according to Figure 6 and Equation (18), and a detailed description can be found in [12]. All internal streams determined with Figure 9 are shown in Figure 10a.

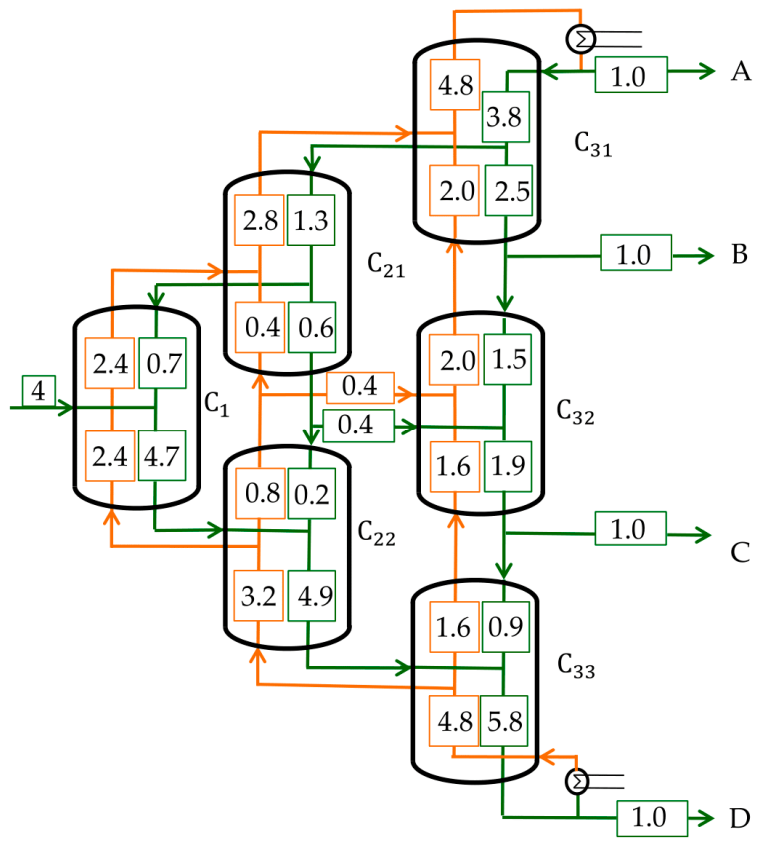

(a)

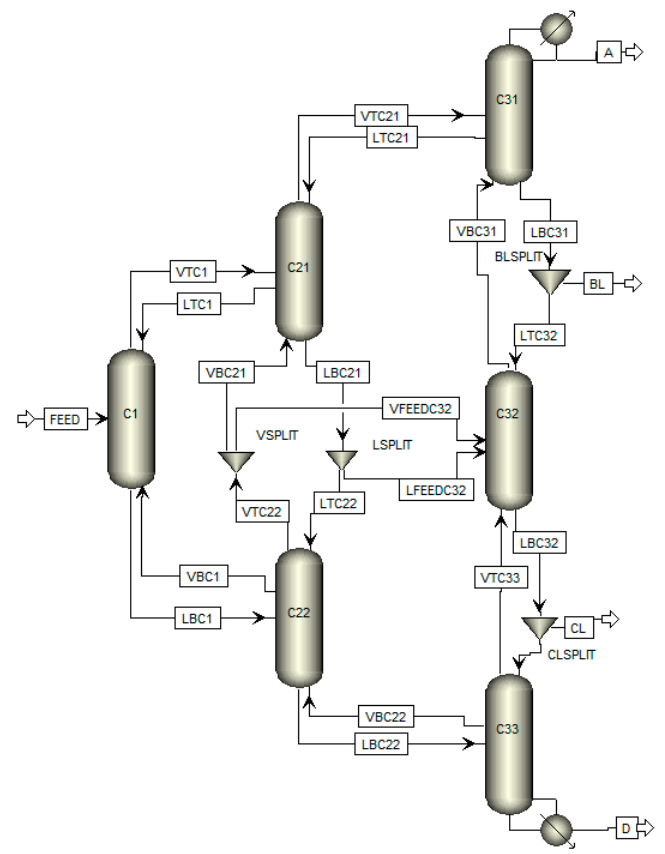

(b)

Figure 10. Six-column Petlyuk arrangement for the separation of 3-Metylhexane (A)/Toluene (B)/Ethylbenzene (C)/1-Methyl-3-Ethylbenzene (D). (a) Internal streams calculated with Figure 9 indicated as numbers in $\mathrm{kmol} / \mathrm{h}$, where green indicates liquid streams and orange vapor streams; and (b) model implemented in Aspen Plus ${ }^{\circledR}$ for rigorous simulation. 
The simulation was performed as a six-column Petlyuk arrangement with liquid side streams implemented in Aspen Plus ${ }^{\circledR}$, as shown in Figure 10b. The column model RadFrac was used due to several reasons. First, columns without a condenser and reboiler are necessary. Second, some of the columns have to be fed with more than one feed stream. Third, the results are needed for each column stage. All three points are not available in the other models as DSTWU. For each column, 50 theoretical stages were assumed, which approximates an infinite number of stages. Feed streams from the pre-sequent columns always enter at the middle of the column at stage 25 . To calculate thermodynamics, NRTL was used since all binary systems were fully defined.

Comparing the top streams of $C_{22}$ with the bottom streams of $C_{21}$ results in a liquid, as well as vapor stream, leaving the two columns to $C_{32}$. The streams in the opposite direction are neglected. Two splitters (LSPLIT and VSPLIT) were implemented to regulate the distribution of the streams. The values that were used for initialization are shown in Table 3.

Table 3. Initial values used for rigorous simulation in Aspen Plus ${ }^{\circledR}$.

\begin{tabular}{|c|c|c|c|c|c|c|}
\hline Variable & Unit & Value & $z_{A}(\mathrm{~mol} / \mathrm{mol})$ & $z_{B}(\mathrm{~mol} / \mathrm{mol})$ & $z_{C}(\mathrm{~mol} / \mathrm{mol})$ & $z_{D}(\mathrm{~mol} / \mathrm{mol})$ \\
\hline VBC22 & $\mathrm{kmol} / \mathrm{h}$ & 3.20 & 0.00 & 0.00 & 0.42 & 0.58 \\
\hline LTC21 & $\mathrm{kmol} / \mathrm{h}$ & 1.40 & 0.69 & 0.31 & 0.00 & 0.00 \\
\hline LTC1 & $\mathrm{kmol} / \mathrm{h}$ & 0.60 & 0.58 & 0.32 & 0.11 & 0.00 \\
\hline VBC1 & $\mathrm{kmol} / \mathrm{h}$ & 2.40 & 0.00 & 0.20 & 0.36 & 0.44 \\
\hline A (distillate C31) & $\mathrm{kmol} / \mathrm{h}$ & 1 & & & & \\
\hline$R^{B}$ at $\mathrm{C} 33$ & 1 & 4.8 & & & & \\
\hline$B L S P L I T=\frac{B L}{L B C 31}$ & 1 & 0.40 & & & & \\
\hline$C L S P L I T=\frac{C L}{L B C 32}$ & 1 & 0.52 & & & & \\
\hline$L S P L I T=\frac{L F E E D C 32}{L B C D 1}$ & 1 & 0.63 & & & & \\
\hline$V S P L I T=\frac{V F E E D C 32}{V T C 22}$ & 1 & 0.50 & & & & \\
\hline
\end{tabular}

With the initial values shown in Table 3, a converging simulation was obtained. The purities of the components are shown in Equation (24).

$$
\begin{gathered}
x_{A}^{A}=99.9 \% \\
x_{B}^{B L}=93.7 \% \\
x_{C}^{C L}=93.3 \% \\
x_{D}^{D}=98.9 \%
\end{gathered}
$$

The mixture was separated successfully even though the product streams are not of high purity. This is caused by the three assumptions on which the Underwood method is based. These are constant molar flows, an infinite number of stages, and constant relative volatilities. The latter one was found to deviate up to $30 \%$ in terms of the values in the feed stream. Additionally, the molar flows were found not to be constant. Nevertheless, it is clear that with the short-cut initialization method of $V_{\min }$ diagrams simulation can be performed successfully.

The temperature profile of the process is shown in Figure 11. Stage numbering starts at the top of the columns.

As typically seen for distillation columns, the temperature increases from the bottom to the top of the column. For the special case of Dividing Wall Columns, the temperature splits on different sides of the dividing walls. In the middle section around the feed stage, the temperature is the lowest in the prefractionating section (column $C_{1}$ ). The temperature has a higher value behind the first dividing wall (lower part of $C_{21}$ and upper part of $C_{22}$ ). A further increase can be noted in the last column row at the same stage as the feed (column $C_{32}$ ). At the liquid and vapor splits, the temperature profiles cross (for example $C_{21}-C_{1}$ at stage 50).

With this example, it was shown that the initialization of rigorous simulations with $V_{\min }$ diagrams is possible. 


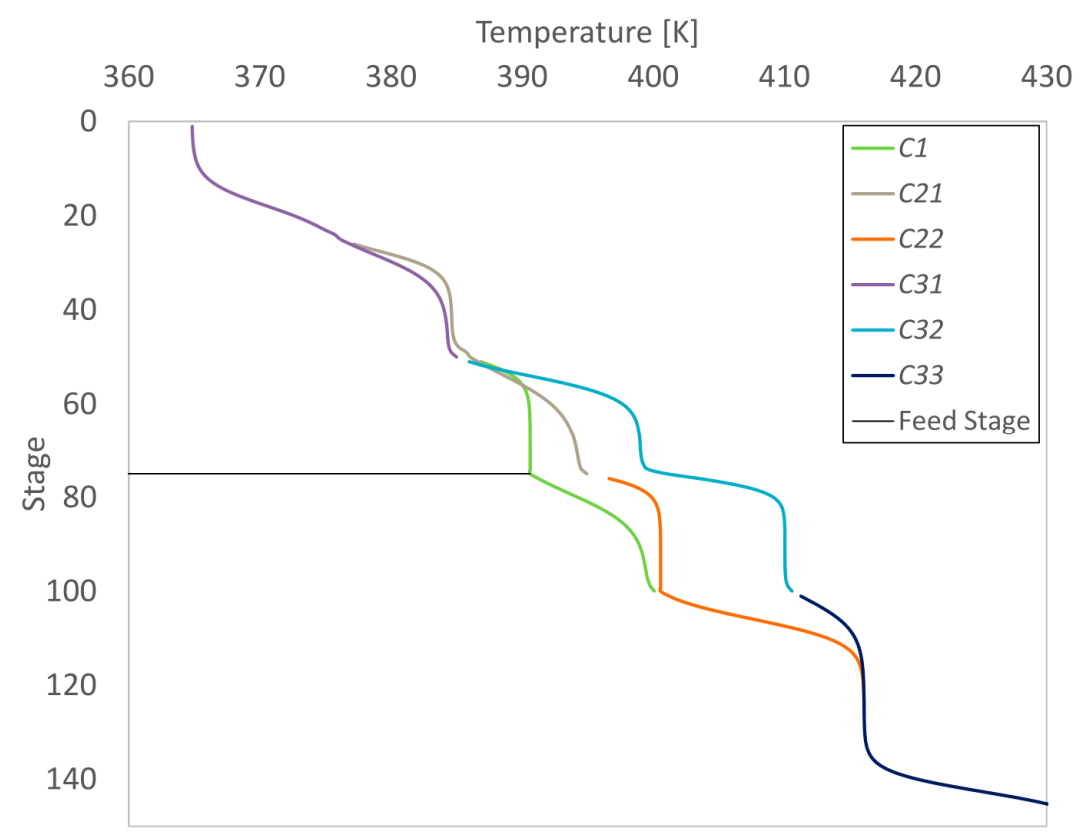

Figure 11. Temperature profile of the separation of 3-Metylhexane/Toluene/Ethylbenzene/1-Methyl-3Ethylbenzene in a conventional mDWC.

\section{Summary}

The main goal of this work was to present the $V_{\min }$ diagram as a robust tool to initialize rigorous simulations of mDWCs. First, the $V_{\min }$ method was presented, showing that the calculation procedure is always the same, independent of the number of components and the column configurations. First, the standard $V_{\min }$ diagram for one column is calculated. In order to do this, the first step is to determine the feed equation which is used to determine the common Underwood Roots of the original feed stream (Section 3.2.1). Second, the active roots are used to calculate the minimum vapor streams (Section 3.2.2). For every additional component, there is one equation and one unknown one, which raises the computational effort but does not change the general procedure. Afterwards, the equivalent column sequence describing the $\mathrm{mDWC}$ has to be evaluated. In any case, the $V_{\min }$ diagram calculated with the initial feed properties is at least valid in the prefractionator. If one of the columns does not work at its preferred split, the diagram changes for the subsequent columns. New roots are calculated by determining a new feed equation around the respective column (Section 4.3), which are again used to calculate the minimum vapor amount (Section 3.2.2). In the end, it could be proven that initialization of rigorous simulations with $V_{\min }$ diagrams in a robust and fast way is possible (Section 5).

In summary, the $V_{\min }$ diagram is a simple short-cut method that can effectively be used to initialize rigorous simulations, which in the end, can be used to construct complex multiple Dividing Wall Columns.

Author Contributions: L.-M.R.: Investigation, Formal Analysis, Software, Methodology, Visualization and Writing-Original Draft; U.P.: Validation, Writing-Review and Editing; T.G.: Conceptualization, Project Administration, Resources, Writing-Review and Editing.

Conflicts of Interest: The authors declare no conflict of interest.

\section{Symbols}

$\begin{array}{lll}\text { Symbol } & \text { Description } & \text { Unit } \\ B & \text { Molar bottom stream } & \mathrm{kmol} / \mathrm{h} \\ D & \text { Molar distillate stream } & \mathrm{kmol} / \mathrm{h} \\ F & \text { Molar feed stream } & \mathrm{kmol} / \mathrm{h}\end{array}$




$\begin{array}{lll}K_{i} & \text { Distribution ratio of component i } & 1 \\ L & \text { Molar liquid stream } & \mathrm{kmol} / \mathrm{h} \\ n & \text { Number of components } & - \\ q & \text { Liquid fraction of feed } & 1 \\ R^{B} & \text { Boilup ratio } & 1 \\ r_{i} & \text { Recovery of component i } & 1 \\ R^{T} & \text { Reflux ratio } & 1 \\ V & \text { Molar vapor stream } & \mathrm{kmol} / \mathrm{h} \\ x_{i} & \text { Molar fraction of component i in liquid phase } & 1 \\ y_{i} & \text { Molar fraction of component i in vapor phase } & 1 \\ z_{i} & \text { Molar fraction of component i in the feed } & 1 \\ \alpha_{\mathbf{i}} & \text { Relative volatility of component i (Equation (2)) } & 1 \\ \theta & \text { Common Underwood Root } & - \\ \Phi & \text { Actual Underwood Root in rectifying section/top of column } & - \\ \Psi & \text { Actual Underwood Root in stripping section/bottom of column } & -\end{array}$

\title{
Subscripts
}

$\begin{array}{ll}\text { Subscript } & \text { Description } \\ \text { A } & \text { Component A } \\ \text { B } & \text { Component B } \\ \text { C } & \text { Component C } \\ \text { D } & \text { Component D } \\ \text { dist } & \text { Distributing } \\ \text { HB } & \text { Feed component with highest boiling temperature } \\ \text { i } & \text { Component } i \\ \text { min } & \text { Minimum } \\ * & \text { Active } \\ \text { B } & \text { Bottom of column } \\ \text { Ci } & \text { Column number i } \\ \text { F } & \text { Feed stream } \\ \text { T } & \text { Top of column }\end{array}$

\section{Abbreviations}

\author{
Abbreviation Description \\ DWC Dividing Wall Column \\ mDWC Multiple Dividing Wall Column
}

\section{References}

1. Kiss, A.A. Advanced Distillation Technologies. Design, Control, and Applications, 1st ed.; Wiley: Chichester, UK, 2013.

2. Dejanović, I.; Matijašević, L.; Olujić, Ž. Dividing Wall Column-A breakthrough towards sustainable distilling. Chem. Eng. Process. Process Intensif. 2010, 49, 559-580. [CrossRef]

3. Staak, D.; Grützner, T.; Schwegler, B.; Roederer, D. Dividing Wall Column for industrial multi purpose use. Chem. Eng. Process. Process Intensif. 2014, 75, 48-57. [CrossRef]

4. Dejanović, I.; Matijašević, L.; Halvorsen, I.J.; Skogestad, S.; Jansen, H.; Kaibel, B.; Olujić, Ž. Designing four-product Dividing Wall Columns for separation of a multicomponent aromatics mixture. Chem. Eng. Res. Des. 2011, 89, 1155-1167. [CrossRef]

5. Yildirim, Ö.; Kiss, A.A.; Kenig, E.Y. Dividing Wall Columns in chemical process industry: A review on current activities. Sep. Purif. Technol. 2011, 80, 403-417. [CrossRef]

6. Triantafyllou, C.; Smith, R. The Design and Optimisation of Fully Thermally Coupled Distillation Columns. Chem. Eng. Res. Des. 1992, 118-132.

7. Kaibel, G. Distillation columns with vertical partitions. Chem. Eng. Technol. 1987, 10, 92-98. [CrossRef] 
8. Halvorsen, I.J.; Skogestad, S. Minimum Energy Consumption in Multicomponent Distillation. 1. Vmin Diagram for a Two-Product Column. Ind. Eng. Chem. Res. 2003, 42, 596-604. [CrossRef]

9. Halvorsen, I.J.; Skogestad, S. Minimum Energy Consumption in Multicomponent Distillation. 2. Three-Product Petlyuk Arrangements. Ind. Eng. Chem. Res. 2003, 42, 605-615. [CrossRef]

10. Halvorsen, I.J.; Skogestad, S. Minimum Energy Consumption in Multicomponent Distillation. 3. More Than Three Products and Generalized Petlyuk Arrangements. Ind. Eng. Chem. Res. 2003, 42, 616-629. [CrossRef]

11. Underwood, A.J.V. Fractional Distillation of Multicomponent Mixtures. Ind. Eng. Chem. 1949, 41, $2844-2847$. [CrossRef]

12. Fidkowski, Z.T.; Agrawal, R. Multicomponent thermally coupled systems of distillation columns at minimum reflux. AIChE J. 2001, 47, 2713-2724. [CrossRef]

13. Ge, X.; Liu, B.; Yuan, X.; Liu, B. Simplifying and synthesizing practical four-product Dividing Wall Column configurations. Chem. Eng. Res. Des. 2017, 125, 433-448. [CrossRef]

14. Koehler, J.; Poellmann, P.; Blass, E. A Review on Minimum Energy Calculations for Ideal and Nonideal Distillations. Ind. Eng. Chem. Res. 1995, 34, 1003-1020. [CrossRef]

15. Mazzotti, M. Underwood's Equations: Derivation: Multicomponent Distillation Column Design; ETH Zürich: Zürich, Switzerland; Available online: http://webarchiv.ethz.ch/hyper-tvt/ppt_pdf/underwood.pdf (accessed on 1 May 2018).

16. Halvorsen, I.J. Minimum Energy Requirements Minimum Energy Requirements in Complex Distillation Arrangements. Ph.D. Thesis, Norwegian University of Science and Technology, Trondheim, Norway, 2001.

17. Asprion, N.; Kaibel, G. Dividing Wall Columns: Fundamentals and recent advances. Chem. Eng. Process. Process Intensif. 2010, 49, 139-146. [CrossRef]

18. Ghadrdan, M. Optimal Operation of Kaibel Columns. Ph.D. Thesis, Norwegian University of Science and Technology, Trondheim, Norway, 2014.

19. Halvorsen, I.J.; Skogestad, S.; Dejanović, I.; Matijašević, L.; Olujić, Ž. Multi-Product Dividing Wall Columns: A Simple and Effective Assessment and Conceptual Design Procedure. In Proceedings of the PRES 2011, 14th Conference on Process Integration, Modelling and Optimisation for Energy Saving and Pollution Reduction, Florence, Italy, 8-11 May 2011; Klemeš, J.J., Ed.; AIDIC: Milano, Italy, 2011; pp. 611-616. 